\title{
Battlefield Advanced Trauma Life Support
}

\section{Chapter 10 \\ Spine and Spinal Cord Injuries}

\section{AIM}

1001. On successfully completing this topic you will be able to:

- Assess spinal injury.

- Apply immobilisation techniques.

- Apply the principles of the management of casualties with spinal injury.

\section{PRINCIPLES OF EVALUATION AND TREATMENT}

1002. In the context of this publication, spinal injuries refer to injuries to the bony spinal column or to the spinal cord, or to both. The principles involved in the evaluation and treatment of spinal injuries are:

- There can be an injury to the bony spine without injury to the spinal cord, but the potential for spinal cord injury is always present.

- Undue manipulation or movement can cause additional spinal cord injury and worsen the prognosis.

- Careful handling of the casualty is essential. When the mechanism of injury dictates, always assume a spinal injury and keep the spine immobilised until you have excluded injury by examination and X-ray.

1003. With all spinal injuries or suspected spinal injuries the aim is:

- To immobilise and protect the spine and the spinal cord.

- To prevent neurological deterioration due to hypoxia, underperfusion or movement of the spinal cord.

- To prevent complications of paralysis.

\section{HISTORY}

1004. The mechanism of injury, the casualty's neurological status, other pertinent physical signs and the potential for further injury, must be recorded at each role of medical care. This will allow recognition of any deterioration or improvement in the casualty's condition at each stage of the evacuation chain.

\section{Mechanism Of Injury}

1005. Is is a blunt injury or a penetrating injury?
- A blunt injury could be due to, for example, deceleration in a road traffic accident, injury to crew in a disabled armoured vehicle, blast, trench cave-in or a parachuting accident.

- A penetrating injury could be due to gunshot, fragments or stabbing. These carry a high mortality especially when caused by high energy-transfer (see paragraph 0416).

\section{Neurological Status}

1006. This will be important in the management of the injury at later roles of medical care. You must always be highly suspicious of a possible cervical spine injury in an unconscious casualty.

\section{EXAMINATION AND ASSESSMENT}

1007. Ideally, before the start to examine and assess casualties with suspected spinal injuries you must immobilize the whole spine in the neutral position. This is the gold standard in peacetime and should remain so whenever possible in wartime, although it may be necessary to compromise on this standard because of the tactical or physical situation on the battlefield. For example, it may be necessary to extract a casualty hurriedly from a vehicle that is ablaze or under attack. It is also difficult to achieve the ideal when dealing with a casualty singlehanded.

1008. There are three groups to consider:

- The conscious casualty without paralysis. You must assume that a casualty with a blunt injury above the clavicle has a spinal injury.

- The conscious casualty with paralysis. This casualty can localize pain, identify sensory loss and demonstrate motor weakness. $\mathrm{Be}$ aware that paralysis and sensory loss may mask intra-abdominal or lower limb injuries.

- The unconscious casualty. About $15 \%^{1}$ of unconscious casualties have some form of neck injury. Clinical findings that suggest spinal cord injury include:

- Flaccid areflexia of limbs.

- A lax anal sphincter.

- Diaphragmatic breathing.

- Response to pain above the clavicle but not below.

- Flexion - but no extension - at the elbow. 
- Hypotension and bradycardia without hypovolaemia.

- Prispism (unopposed parasymathic drive).

\section{Spinal Assessment}

1009. Carefully palpate the spine for localised tenderness, a palpable gap between the spinous processes and localised swelling. At some stage during the examination, you must perform a careful log-roll to look for bruising and deformity.

\section{Neurological Assessment}

1010. Examine the casualty for motor strength and weakness, sensory changes and altered reflexes:

- Autonomic nervous system. You will recognise autonomic dysfunction by loss of bladder and anal sphincter control and the presence of priapism. Bradycardia and hypotension may be present.

- Incomplete neurological damage. This may present in a variety of ways. Whichever way it presents, there is always evidence of motor or sensory function below the level of injury. In such cases, there may be sparing of sensation in the sacral area and the anal sphincter tone can be normal. Neurological recovery is likely in such cases.

- Complete cord lesion. There is no evidence of neurological function below the level of injury; the prognosis is poor.

\section{Neurogenic shock}

1011. This is the term for hypotension with high thoracic or cervical cord injuries. Hypotension results from destruction of the sympathetic pathways with loss of vasomotor tone and loss of sympathetic drive to the heart. There can also be bradycardia because of the unopposed parasympathetic (vagal) effect on the heart.

\section{Spinal shock}

1012. This is a neurological condition occurring shortly after a spinal cord injury. It results in limb flaccidity and areflexia, a flaccid bladder and loss of drive by the sympathetic nervous system. The condition is variable but normally lasts about six weeks.

\section{Assessment Of Other Systems Cardiovascular}

1013. Central. Asystole can occur from stimulation of the unopposed action of the vagus, for example, by pharnyngeal suction and nasogastric or endotracheal intubation. It is prevented by giving atropine $0.5 \mathrm{mg}$ intravenously. A bradycardia of $60 / \mathrm{min}$ is usual in the high, isolated cord injury.

1014. Peripheral. Paralysis of the sympathetic system results in hypotension (see paragraph 1011). The casualty feels warm and well perfused due to peripheral vasodilatation. Do not forget that the most common cause of hypotension is due to blood loss and hypovolaemia often co-exists with spinal injury. Always assess and treat for hypovolaemic shock first.

Respiratory

1015. If the injury is above the fourth vertebra there is no respiration. With injuries between the fourth cervical and the twelfth thoraic vertebrae there will be intercostal paralysis and, depending on the level, there may be only diaphragmatic breathing.

1016. Complicating factors are rib fractures, flail chest, pulmonary contusion, haemopneumothorax and aspiration pneumonitis. You must vigorously address these problems by providing ventilatory support, chest drainage and, if the casualty can feel pain, analgesia.

\section{Abdomen}

1017. Inability to feel pain may mask serious intra-abdominal injury. The only symptom pointing to an intra-abdominal problem may be referred shoulder-tip pain. Ileus is usual in a paralysed casualty; you should decompress the stomach with a nasogastric tube. This will also minimise dangerous silent regurgitation and aspiration of gastric contents.

\section{Locomotor}

1018. Pelvic injuries may be masked by a spinal cord injury and, if present, may merit a higher priority in the management of the casualty.

1019. Lower limb injuries are easily missed in the absence of sensation. Remember that early and correct management of upper limbs injuries may have a profound effect on the eventual mobility of a quadriplegic.

\section{Skin}

1020. In a high cord lesion, temperature control function is lost and the casualty may become hypothermic or hyperthermic.

\section{Bladder}

1021. Casualties with spinal cord injury and urinary retention need continuous catherer drainage. The recording of urinary output is a good monitor of response to resuscitation if there are other injuries requiring treatment by intravenous fluid administration.

\section{Treatment}

Follow the primary survey: the $A B C D$ E routine

1022. Treat as follows:

- All casualties:

- Ensure in-line immobilization of the whole spine by any means available, a combination of semi-rigid collar, sandbags and tape and backboards, is ideal.

- Correct any life-threatening conditions found in the primary survey.

- Continue immobilisation until you are certain there is no spinal injury. 
- Conscious casualties with paralysis:

- Maintain mean arterial pressure at 90 $\mathrm{mmHg}$ (a systolic pressure of 120 $\mathrm{mmHg}$ ), with intravenous infunction if necessary. If not contraindicated by the presence of a head injury, a head down position will aid venous return.

- Insert a nasogastric tube under cover of intravenous atrophine (see paragraph 1013).

- Insert a urinary catheter and put this on continuous drainage.

- Remove all hard objects and ensure the backboard or stretcher is well padded.

- Unconscious casualties:

- Keep the back and neck straight.

- In order to protect the airway it may be appropriate to evacuate the casualty in the three-quarter prone position. If this is done, a supporting pillow or blanket under the head will reduce the risk of lateral neck flexion.

- Maintain three-point stability at the pelvis, knee and shoulder.

- Keep one hemidiaphragm clear of the ground.

- If anaesthetic support is available, intubation and ventilation is preferred and should be established before the casualty is transferred.

- Remember: although good spinal immobilization is ensured by using back-boards, they are not a good carrying device as the casualty is balanced on seven bony points - the occiput, scapulae, ischial tuberosities and the calcanei. These bony points are vulnerable to pressure sores if evacuation time is protracted.

\section{EVACUATION}

1023. Before evacuation ensure that:

- The airway is secured, the casualty is ventilating with or without support and well oxygenated.

- The casualty is adequately immobilized and secured, is well padded and not in any danger from hard objects. Do not use halter traction.

- Sufficient drugs, such as atrophine, are available for the journey.

1024. During evacuation ensure that:

- Ventilation and oxgenation remain adequate.

- Immobilisation is maintained.

- Intravenous infusion lines, urinary catherers and nasogastric tubes remain secure.

- In-transit escorts are capable of managing the casualty.

\section{SUMMARY}

- Deal with life-threatening conditions but avoid any movement of the spinal column.

- Establish adequate immobilisation and maintain it until you are certain there is no spinal injury.
- Record history and clinical findings as a base-line for changes in the neurological status.

- Obtain X-rays when facilities are available.

- Evacuate.

\begin{tabular}{|c|}
\hline Chapter 11 \\
Limb Injuries \\
\hline
\end{tabular}

\section{AIM}

1101. On successfully completing this topic you will be able to:

- Identify life-threatening limb injuries

- Identify limb-threatening injuries

- Outline priorities in the management of limb injury at different roles of medical care.

\section{PRINCIPLES OF \\ MANAGEMENT}

Primary Survey And Resuscitation

Remember the A B C D E routine

1102. The main considerations in the primary survey of limb injury are:

- Control of bleeding, usually by direct pressure or application of a tourniquet.

- Recognition of long-bone fractures and their immediate management - the application of splintage or traction.

- Recognition of covert vascular injury. 1103. The aim is obvious: look for lifethreatening or potentially life-threatening blood loss and stop it.

Secondary Survey

1104. You must examine the limbs in detail and evaluate all limb injuries. In examination you must:

- Record the presence of wounds, swelling or limb deformity.

- Assess limb circulation; note perfusion by checking capillary return. This should not exceed two seconds; note the colour and temperature of the skin and presence of peripheral pulses (absent in $70 \%$ of vascular injuries).

- Record any neurological disability following nerve damage.

\section{Definitive Care}

1105. Clean and dress wounds with firm sterile dressings. Continue to control haemorrhage. Correct deformities to relieve pain and to protect the circulation. Splint and immobilise fractures. Application of a traction splint has the following effects:

- Reduction of haemorrhage (see Fig. 11.1).

- Reduction of pain

- Prevention of further soft tissue injury

- Reduces the incidence of fat embolism.

\section{ASSESSMENT OF LIMB INJURIES \\ History}

1106. An accurate picture of the 


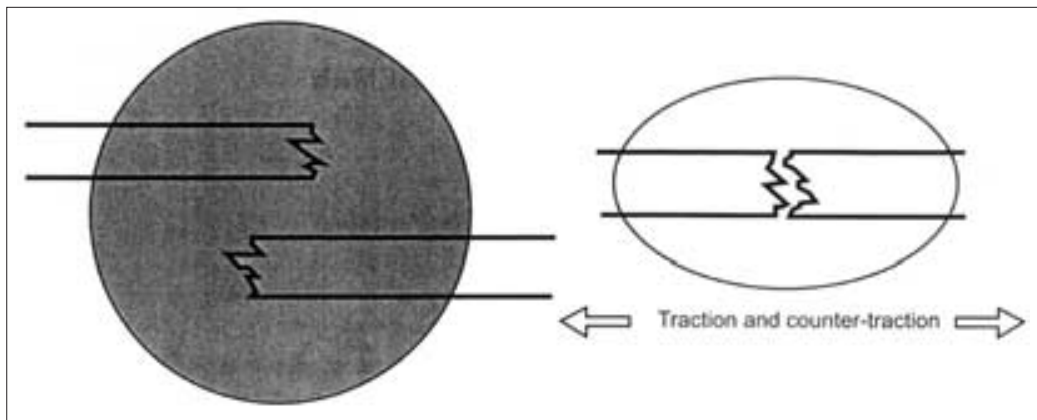

Eliptical area of surrounding tissue produced by traction, holds less bilood and tissue fluid than a spherical area

Fig. 11.1 Traction number of severe limb injuries, or injuries in other areas contributing to blood loss. It is on record that up to $10 \%$ of battlefield casualties with limb injuries bleed to death unnecessarily. Do not let this happen. Remember, the majority of haemorrhage from limb injuries is compressible or, controllable by correct use of a tourniquet. To a lesser extent, haemorrhage from pelvic fractures is also compressible.

\section{Limb Threatening Injuries}

1109. Most limb-threatening injuries are related to vascular injury, particularly if the injury is proximal to the knee or elbow. Fractures or fracture-dislocations around the knee or elbow are commonly associated with injury to the popliteal and brachial artery respectively. Crush injuries may result in later amputation, because of cell damage. An unrecognised compartment syndrome will result in permanent disability. Open fractures will lead to severe complications if ignored.

\section{Associated Injuries}

1110. It is important to realize that the casualty may have multiple injuries. Knowledge of the cause of the injury is important: for example, a fall from a height can result in vertebral fractures or fractures of the calcanei as well as long bone fractures. Some fractures are not easy to detect and are found only after repeated examination. Do not forget the possibility of a cervical spine injury in falls from a height.

\section{Assessment Of Blood Loss}

1111. Limb injuries play an important role when estimating blood loss. With open wounds the loss may be evident. Equally, the overall condition of the casualty may be the clue to covert haemorrhage. A closed fracture of the femoral shaft may easily result in the loss of two litres into the surrounding tissues. The summation of the effect of a number of such injuries is evident when the signs of hypovolaemic shock are detected.

- Move. Check all the limbs for active movements where possible. With an unconscious casualty, test each limb passively for range of movements.

- Fracture assessment. Is the fracture open or closed? Any fracture with a would adjacent to it must be assumed to be an open fracture. Note any bone protrusion remember that reduction of potentially contaminated protruding bone is likely when splints are applied. Surgical toilet of the bone may be required later.

\section{Life Threatening Limb Injuries}

1107. Life-threatening injuries include:

- Complete or incomplete traumatic amputations.

- Multiple long bone fractures.

- Major pelvic injuries.

1108. The threat to life is caused by blood loss; this threat is even greater if there are a

\section{Remember the four areas for occult blood loss}

- Chest

- Abdomen

- Pelvis and retroperitoneum

- Long-bone fractures

\section{Assessment Of Dislocations}

1112. Dislocations and fracture-dislocations are difficult to distinguish without X-rays. Dislocations are extremely painful when attempts are made to move the joint and this helps early recognition. Such early recognition can allow prompt reduction especially if there is altered blood supply to the limb, for example in posterior dislocation of the knee occluding the popliteal artery. 


\section{Assessment Of Vascular Injury}

1113. A major vascular injury may be suggested by:

- Obvious arterial or venous haemorrhage from the wound.

- An expanding haematoma.

- Absent distal pulses (70\%).

- Delayed capillary return.

- Differing skin colour and temperature compared with the contralateral limb.

- Increasing pain at the site of the injury.

- Decreasing sensation.

Repeated assessment of the circulation is necessary especially when the casualty arrives at a new role of medical care.

\section{Assessment Of Nerve Injury}

1114. Some injuries are often associated with neurological damage, for example a dislocated hip and sciatic nerve injury, or a dislocated elbow and median nerve injury. Confirmation can be obtained by the presence of alteration of sensation or motor power, or both. If you detect an inappropriate nerve injury, you must consider the possibility of a cerebral or spinal injury.

\section{Compartment Syndrome}

1115. Untreated, this will lead to rapid loss of a limb or permanent disability. Prompt recognition and emergency surgery are needed. Causes include crush injuries or prolonged limb compression, open or closed fractures, the restoration of circulation to an ischaemic limb and tight plasters or dressings. Compartment syndrome occurs when the interstitial pressure in a fascial compartment exceeds the capillary pressure as a result of haemorrhage or oedema within the involved compartment. Initially, venous flow stops and as the pressure increases the arterial supply also stops. Ischaemia of nerves and muscles occurs with rapid and irreversible damage. The distal pulses may be present throughout. The compartments most commonly affected are the anterior tibial compartment and the flexor compartment of the forearm. The main presenting sympton is severe pain in an injured limb that is adequately immobilised. The pain is aggravated by passive stretching of the muscles in the involved compartment. Active movements are absent. The compartments are swollen, tense, tender and the distal sensation may be altered.

The presence of a distal pulse does note exclude a compartment syndrome.

\section{Crush Syndrome}

1116. This causes damage to muscle cells releasing potassium and myoglobin into the circulation. High levels of potassium in the blood can cause cardiac arrest. Myoglobin blocks renal tubules, leading to renal failure. Before releasing a trapped injured limb you must give intravenous crystalloid in large volumes. To avoid renal failure, intravenous fluids should be given in sufficient volume to produce a minimum of $100 \mathrm{ml}$ of urine per hour. An ECG monitor, if available, may give warning of hyperkalaemia (broad bizarre complexes; tented T-wave) ${ }^{1}$.

\section{MANAGEMENT OF LIMB INJURIES \\ Fractures}

1117. With an open fracture, control haemorrhage by direct pressure, firm compression, bandaging and elevation of the limb. This will cope with most bleeding. Pressure points, which are difficult to compress, are rarely used. Remove gross contamination, such as earth and bits of clothing and clean the wound with copious irrigation before applying a dry sterile compression dressing. Splint severe soft tissue wounds to relieve pain and to control haemorrhage. Describe the wound in the notes to avoid repeated disturbance of the dressing before definitive treatment. Repeated wound inspection increases the risk of infection. The fracture should then be treated as for any other fracture. Protruding bone should be carefully reduced back into the wound, usually by the application of traction.

\section{Emergency Amputations}

1118. A mangled limb with no prospect of reconstruction should be removed. Such a limb will usually be attached only by a few remnants of skin or other soft tissues. To leave it, especially in the presence of other injuries, may endanger the casualty's life. In removing the limb, preserve as much healthy skin, fascia and muscle as possible. You can amputate through the fracture site. Transfix the major blood vessels and ligate them with strong suture material. Divide nerves and allow them to retract. Leave flaps open, firmly bandage the stump and apply a plaster splint. The use of a tourniquet may be considered to reduce blood loss during the procedure. Skin from the amputated part may subsequently be used for grafting. If the part is not too mangled and the casualty evacuation is not delayed, send it with the casualty to the surgeon. 


\section{Dislocations}

1119. Reduce all dislocations at the earliest opportunity. They are often relatively easy to reduce soon after injury. Remember that they are painful injuries. Reduction under Entonox, when available, avoids the need for continued monitoring after sedation. Ketamine in analgesic doses provides excellent analgesia for five to ten minutes and will not depress respiration like morphine. Immobilise the joint after reduction but check the distal circulation before you do so.

\section{Vascular Injuries}

1120. If you suspect a major vessel injury you must control haemorrhage from the wound, dress the wound and splint the limb. Early evaculation for further investigation and definitive treatment is necessary if the limb is to be saved.

\section{Compartment Syndrome}

1121. If you suspect this condition and the limb is in plaster or has circumferential dressings, split them completely down to the skin and open them widely. If symptoms do not improve within 15 minutes, any dressings overlying open wounds should be removed and the underlying muscle examined. Its colour should like like raw, red meat; if it does not, suspect compartment syndrome. Such a limb requires urgent fasciotomy.

Do not hesitate to remove plaster casts and circumferential dressings.

\section{Immobilisation}

1122. Adequate splintage will relieve pain and help to control haemorrhage. The circulation and soft tissues will also be protected.

\section{Splinting Limbs}

1123. Support the hand with a wool and crépe bandage. Immobilise injuries of the forearm or upper arm with padded splints or plaster, together with a broad arm sling. Keep the elbow at a right angle. If only one leg is injured, splint it to the good limb, together with padded external splints and bandages. A traction splint should be used for fractures of the femur, but not if there is an ankle fracture on the same side. Obvious deformity and rotation of long bones should be corrected before splinting - this relieves pain, protects the circulation and makes splinting easier.

\section{The Tourniquet}

1124. The Samway anchor tourniquet is available at all roles of medical care but should only be used when other measures fail or compression is difficult. For example, it will be more appropriate to immediately apply a tourniquet to a trapped limb when access for adequate compression is impossible. A tourniquet may also be necessary when completing a traumatic amputation. A tourniquet must be applied effectively above the level of haemorrhage to stop arterial flow; it should be released as soon as possible. But, remember you have applied the tourniquet to save the casualty's life. Ischaemic damage to a limb is rare if a tourniquet is left for up to one hour. If it is feasible to control haemorrhage by direct compression, release the tourniquet. If haemorrhage is not controlled, or not controllable, by compression, re-apply the tourniquet. If transfer of the casualty is delayed, repeat this process every hour and discard the use of the tourniquet as soon as haemorrhage is controlled by compression.

1125. Incorrectly applied, the tourniquet will increase venous pressure and aggravate, rather than control, haemorrhage. Keep at the forefront of your mind the fact that, inappropriate or incorrect use of a tourniquet, may result in unnecessary limb loss. A tourniquet, used correctly and for the right reasons, will save a life.

\section{SUMMARY}

- Manage life-threatening injuries first.

- Dress wounds, align fractures and immobilise.

- Record details.

- Determine priority and evacuate.

\section{Skills Station 8} Limb Splintage

\section{AIM}

The aim of this skills station is to give you the opportunity to practice and demonstrate the proper techniques for immobilising limbs. On completion of this station you will be able to:

- Demonstrate the steps in the control of haemorrhage and the initial management of wounds.

- Demonstrate the application of splints to upper and lower limbs.

- Demonstrate the application of a traction splint to a lower limb.

- Discuss the advantages and dangers in the use of a tourniquet.

\section{EQUIPMENT}

\begin{tabular}{|l|}
\hline Padded wood and Kramer wire splints. \\
Broad arm bandages. \\
Traction splint. \\
Plaster of Paris, wool and gauze. \\
Plaster cutters. \\
Stretcher. \\
Basin and cleaning materials. \\
Samway anchor tourniquet. \\
\hline
\end{tabular}

